\title{
Microlensing search for extrasolar planets: observational strategy, discoveries and implications
}

\author{
Arnaud Cassan ${ }^{1,4} \dagger$ and Takahiro Sumi ${ }^{2,5} \ddagger$ and Daniel Kubas ${ }^{3,4}$ \\ ${ }^{1}$ ARI/ZAH Heidelberg University, Germany \\ ${ }^{2}$ Nagoya University, Japan \\ ${ }^{3}$ ESO, Chile \\ ${ }^{4}$ PLANET/RoboNET Collaborations \\ ${ }^{5}$ MOA Collaboration
}

\begin{abstract}
Microlensing has proven to be a valuable tool to search for extrasolar planets of Jovian- to Super-Earth-mass planets at orbits of a few AU. Since planetary signals are of very short duration, an intense and continuous monitoring is required. This is achieved by groundbased networks of telescopes (PLANET/RoboNET, $\mu$ FUN) following up targets, which are identified as microlensing events by single dedicated telescopes (OGLE, MOA). Microlensing has led to four already published detections of extrasolar planets, one of them being OGLE 2005BLG-390Lb, a planet of only $\sim 5.5 M_{\oplus}$ orbiting its M-dwarf host star at $\sim 2.6$ AU. Very recent observations (May-September 2007) provided four more planetary candidates, still under study, that will double the number of detections. For non-planetary microlensing events observed from 1995 to 2006 we compute detection efficiency diagrams, which can then be used to derive an estimate of the Galactic abundance of cool planets in the mass regime from Jupiters to SubNeptunes.
\end{abstract}

Keywords. Extrasolar planets, Gravitational microlensing

\section{Introduction}

A Galactic microlensing event occurs when a massive compact intervening object (the lens) deflects the light coming from a more distant background star (the source). It leads to an apparent flux brightening (or magnification) of the source star. In a typical scenario, the source belongs to the Galactic bulge, while the lens can be part either of the bulge (2/3 of the events) or the disk (1/3 of the events) population. Mao \& Paczyński (1991) were the first to suggest that microlensing could provide a powerful tool to search for extrasolar planets at distances of a few kpc, provided a continuous monitoring of bulge stars. Since the detection of planets by microlensing does not rely on their light but on their mass, the planetary host mass function basically follows the stellar mass function of the Galaxy, implying that planet hosts are preferably low-mass $\mathrm{K}$ to $\mathrm{M}$ dwarfs.

The relative motion between source, lens and observer ( $\sim 15 \mu \mathrm{as} /$ day $)$ induces a variation of the magnification factor with time, with a typical time scale of $t_{\mathrm{E}} \simeq 40 \times \sqrt{M_{*} / M_{\odot}}$ days, where $M_{*}$ is the lens mass and assuming a source and lens distance of respectively 8.5 and $6.5 \mathrm{kpc}$. The duration of the planetary light curve signal then scales as $t_{\mathrm{p}} \approx 2 \sqrt{q} \times t_{\mathrm{E}}$, where $q$ is the planet-to-star mass ratio, which means few days for a giant planet to only few hours for Neptune- to Earth-mass planets.

$\dagger$ Based on the talk "Microlensing search for extrasolar planets".

$\ddagger$ Based on the talk "Exoplanet Candidates from the MOA-II Microlensing Survey in 2007". 
The microlensing method is remarkable in the sense that it probes a domain in the planet mass-orbit diagram that is mainly out of reach of other techniques, for it is mostly sensitive to Jovian- down to Earth-mass planets (e.g. Bennett \& Rhie 2002) with orbits of $\sim 1-10 \mathrm{AU}$, at several kpc.

\section{Alerts and follow-up of microlensing events}

Microlensing surveys (OGLE \& MOA) currently monitor more than $\sim 10^{8}$ Galactic bulge stars by 1-2 m class telescopes on a daily basis to find and alert microlensing events. The second phase of MOA, MOA-II, carries out survey observations toward the Galactic bulge to find exoplanets via microlensing using a $1.8 \mathrm{~m}$ telescope in New Zealand. We observe our target fields $\left(\sim 50 \mathrm{deg}^{2}\right)$ very frequently (10 to 50 times/night) and analyze data in real-time to issue microlensing alerts. This high cadence is specifically designed to find the short duration time signatures characteristic of planets orbiting the lens star. In 2007, real-time event monitoring started in order to search for planetary signatures in ongoing microlensing events. Each new data point on the light curves is available within 5 minutes after image exposure. During 2007, MOA has detected around 500 microlensing events.

While single alert telescopes are able to identify and follow microlensing planetary candidates, they somehow suffer from gaps in the data coverage. Network of telescopes, as operated by the PLANET/RoboNET or $\mu \mathrm{FUN}$ collaborations perform a "round-theclock" monitoring of a reduced number of selected targets, which significantly increases the planet detection efficiency. For example, with currently five 1m-class telescopes located in Chile, two in South Africa, Australia and Tasmania, as well as using three robotic telescopes, PLANET/RoboNET currently has unequaled capability for covering microlensing events, by minimizing data gaps in which planetary signatures could hide.

Recent efficient and interactive communication between alert and network collaborations has played a major role in improving the ability to quickly focus on suspected planetary signal in many events.

\section{Results}

\subsection{Detections and new planetary candidates}

As of now four extrasolar planets detected by microlensing are reported in the literature (see eg. Rattenbury 2006 for a more detailed review). Among them there are two giants of few Jupiter masses, MOA 2003-BLG-53Lb (Bond et al. 2004) and OGLE 2005-BLG071Lb (Udalski et al. 2005), as well as two Super-Earth-mass planets, OGLE 2005-BLG169Lb (Gould et al. 2006) and OGLE 2005-BLG-390Lb (Beaulieu et al. 2006), the latter being one the lightest ever discovered planets, with only $\sim 5.5 M_{\oplus}$ and a wide orbit of $\sim 2.6 \mathrm{AU}$. This first detection of a cool rocky/icy sub-Neptune mass planet has thus opened a new observing window for the exoplanet field.

Very recent observations (May-September 2007) have revealed four more planetary candidates which are in the process of final analysis and are expected to be published in 2008. These new detections will double the number of detections and should provide a better understanding of the statistical properties of the microlensing planet population and also help to optimize he detection efficiency of the current observational set up (see $3.3)$. 


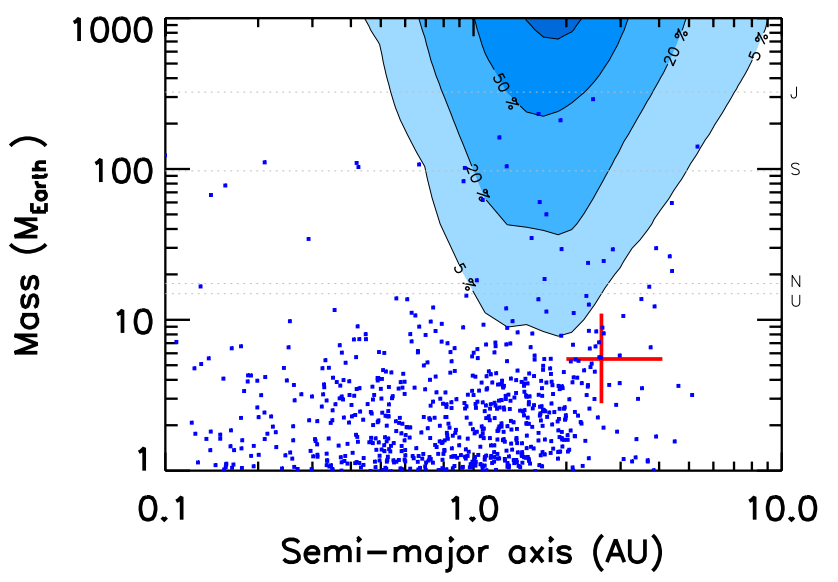

Figure 1. Detection efficiency for an additional planet orbiting OGLE-2005-BLG-390L as a function of its orbital separation and mass. Efficiency contours of 5\%,20\%, 50\% and $70 \%$ are shown. The cross marks the median values for the properties of OGLE 2005-BLG- 390Lb along with $1 \sigma$ confidence intervals and the dashed horizontal lines mark the masses of Jupiter $(\mathrm{J})$, Saturn (S), Neptune (N) and Uranus (U) for comparison. The blue dots represent the predicted final distribution of a seed of $2 \times 10^{4}$ planetary cores around an M-dwarf of $0.2 M_{\odot}$ resulting from a core-accretion model assuming inefficient migration (taken from Fig. $9 \mathrm{~b}$ of Ida \& Lin $(2005))$.

\subsection{Limits on the multiplicity of planetary systems}

Microlensing also allows the direct detection of a multi-planetary system (see the contribution from D. Bennett, this volume), as well as of circum-binary planets.

When only a single planet is detected, it is still possible to put limits on the presence of further planets in the microlensing event, such as in the case of OGLE 2005-BLG390 (Kubas et al. 2007), presented in Fig. 1. Although the detection efficiency depends strongly on the reached peak magnification, a good data coverage can result in significant detection sensitivities even for low peak magnification events. In this particular case one finds that more than $50 \%$ of potential planets with a mass in excess of $1 M_{J}$ between 1.1 and 2.3 AU around OGLE 2005-BLG-390L would have revealed their existence, which was however not observed. For gas giant planets above $3 M_{J}$ in orbits between 1.5 and $2.2 \mathrm{AU}$, the detection efficiency exceeds $70 \%$. Furthermore we find a detection probability for an OGLE-2005-BLG-390Lb-like planet, given an idealization of the microlensing technique, to be around $1-3 \%$. In agreement with current planet formation theories this quantitatively supports the prediction that sub-Neptune mass planets are common around low mass stars.

\subsection{Detection efficiencies}

Apart from the detection of planets a main goal of microlensing observations is to estimate the planet detection efficiency in order to put constraints the Galactic planet abundance. From 42 densely monitored events between 1997 and 1999, PLANET was able to provide the first significant upper abundancy limit of Jupiter- and Saturn-mass planets around M-dwarfs, namely that less than $1 / 3$ of the lens stars have Jupiter-mass companions at orbital radii between 1.5 and $4 \mathrm{AU}$, and less than $2 / 3$ have Saturn-mass companions for the same range of orbital radii, assuming circular orbits (Gaudi et al. 2002). 


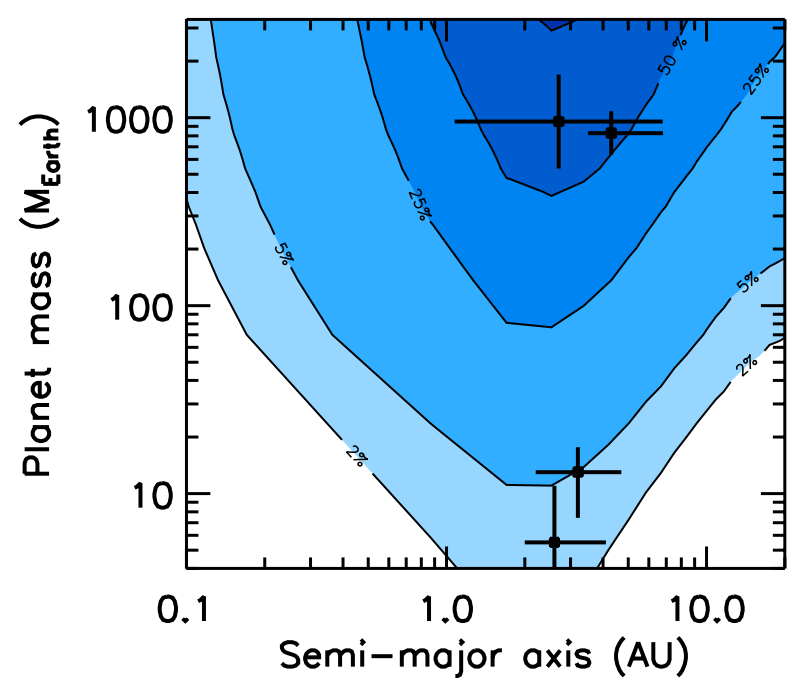

Figure 2. PLANET detection efficiency from the 2004 season (preliminary diagram), as a function of planet mass and orbital separation. The crosses are the detected planets with their parameter error bars.

By using an adequate Galactic model for the distribution of lens masses and velocities (Dominik 2006), we aim to pursue and improve the study, moreover taking into account more than ten years of observations (Cassan et al. 2008). The Fig. 2 shows a preliminary planet detection efficiency diagram, computed from well-covered events of the 2004 season.

\section{Summary and prospects}

Microlensing has proven to be a robust method to search for extrasolar planets at large separations from their parent stars $(\sim 1-10 \mathrm{AU})$. It is sensitive to masses down to the mass of the Earth using ground based telescopes and even capable to detect planets of a few fractions of Earth masses when considering space-based telescope scenarios.

Microlensing is also very well-suited for statistical studies on planet abundance in the Galaxy. In fact, the method is by essence not limited to our close solar neighborhood or to a particular type of host stars.

\section{References}

Mao \& Paczyński, 1991, ApJ, 374, L37

Bennett \& Rhie 2002, ApJ, 574, 985

Bond et al. 2004, ApJ, 606, L155

Udalski et al. 2005, ApJ, 628, L109

Beaulieu et al. 2006, Nature, 439, 437

Gould et al. 2006, ApJ, 644, L37

Rattenbury 2006, Modern Physics Letter A, 21, 919

Kubas et al. 2007, to appear in A\&A (astro-ph/0710.5306)

Gaudi et al. 2002, ApJ, 566, 463

Dominik 2006, MNRAS, 367, 669

Cassan et al. 2008, in preparation 